\title{
Multicenter Validation of a Shortened Gastric-Emptying Protocol
}

\author{
Matthieu Pelletier-Galarneau ${ }^{1}$, Oyebola O. Sogbein ${ }^{1}$, Xuan Pham ${ }^{1}$, Jason $\mathrm{Hao}^{2}$, Jenna Le ${ }^{3}$, Mark D. Strober ${ }^{4}$, \\ Michael L. Middleton ${ }^{4}$, Janusz Kikut ${ }^{2}$, Leonard M. Freeman ${ }^{3}$, and Lionel S. Zuckier ${ }^{1}$ \\ ${ }^{I}$ The Ottawa Hospital, Division of Nuclear Medicine, Ottawa, Ontario, Canada; ${ }^{2}$ University of Vermont Medical Center, Burlington, \\ Vermont; ${ }^{3}$ Montefiore Medical Center, Bronx, New York; and ${ }^{4}$ Baylor Scott \& White Health, Central Texas, Temple, Texas
}

\begin{abstract}
Gastric emptying scintigraphy is considered to be the gold standard for detection of gastroparesis and other disorders of gastric motility; Society of Nuclear Medicine and Molecular Imaging guidelines are predicated on imaging over a period of $4 \mathrm{~h}$, which is inconvenient for patients. Bonta et al. introduced 2-h criteria, which served to shorten the protocol in most patients, with negligible loss of accuracy. We have evaluated the Bonta criteria in a larger multicenter trial encompassing 4 academic institutions. Methods: Retrospective data from 4 academic medical centers were aggregated; 431 patients were included, $105(24.4 \%)$ of whom demonstrated delayed gastric emptying defined by 4 -h gastric retention of more than $10 \%$. Bonta criteria (retention $>65 \%$ is considered abnormal and $<45 \%$ normal; otherwise, proceed to complete examination) were applied to the 2-h data. Sensitivity, specificity, accuracy, and resource use for the Bonta method were calculated. Results based on standard 4-h solid gastric emptying, performed according to current Society of Nuclear Medicine and Molecular Imaging guidelines, served as the gold standard. Results: Retention of $10 \%$ or less was achieved by $6,77,215$, and 326 patients at $1,2,3$, and $4 \mathrm{~h}$, respectively. At 2 h, 261 of 431 patients $(60.6 \%)$ had gastric retention of less than $45 \%$, which according to Bonta would be classified as normal; $62(14.4 \%)$ had gastric retention of more than $65 \%$, which would be classified as delayed emptying; and 108 (25.1\%) had intermediate values requiring further imaging through $4 \mathrm{~h}$. The Bonta criteria yielded a sensitivity, specificity, and accuracy of $92.4 \%, 96.9 \%$, and $95.8 \%$, respectively, superior to any single cutoff point applied to the 2 -h values. The criteria resulted in falsenegative results in $8(1.9 \%)$ patients, 6 of whom were borderlinepositive at $4 \mathrm{~h}$ (gastric retention of $11 \%-14 \%$ ). Using the Bonta criteria, $74.9 \%$ of studies would be terminated by $2 \mathrm{~h}$, decreasing total camera use by $15.7 \%$, from 1,768 to 1,490 images, and the average study duration would be reduced by $20.6 \%$, from 3.1 to 2.5 h. Conclusion: In a multicenter cohort, use of the Bonta criteria shortened the duration of studies in most patients, resulting in an effective compromise between reduced resource use, improved patient convenience, and preserved accuracy.
\end{abstract}

Key Words: gastric emptying; Tougas protocol; Bonta criteria

J Nucl Med 2015; 56:873-876

DOI: 10.2967/jnumed.115.155366

Received Feb. 5, 2015; revision accepted Mar. 31, 2015.

For correspondence or reprints contact: Matthieu Pelletier-Galarneau, Division of Nuclear Medicine, The Ottawa Hospital, General Campus, 501 Smyth Rd., University of Ottawa, Ottawa, Ontario, Canada K1H 8 L6.

E-mail:mattgalarneau@gmail.com

Published online Apr. 16, 2015.

COPYRIGHT (c) 2015 by the Society of Nuclear Medicine and Molecular Imaging, Inc.
$\mathbf{F}$ mon complaint of patients referred to gastroenterologists and affect approximately 1 in 5 adults in the United States, especially young females (1). Most cases of gastroparesis are idiopathic, but delayed gastric emptying has been associated with diabetes, gastrointestinal tract surgery, Parkinson disease, collagen vascular disease, and intestinal pseudoobstruction (2). Gastric emptying scintigraphy (GES) is considered the gold standard for evaluation of patients with suspected delayed gastric emptying (3). In 2000, Tougas et al. published a 4 -h protocol using a solid radioactive meal with a large normal database (4). Consensus recommendations by the American Neurogastroenterology and Motility Society proposed that the Tougas protocol become the standard gastric emptying protocol (3). This protocol is now incorporated into the Society of Nuclear Medicine and Molecular Imaging guidelines for GES (5). Because of its length, the standard protocol can be inconvenient for patients and may be difficult to integrate into the clinical workflow. To address this issue, Bonta et al. retrospectively analyzed 174 patients who underwent GES after ingestion of a standardized meal to determine whether 2-h gastric retention values could predict abnormal or normal results. They found that 2-h values of more than $65 \%$ and less than $45 \%$, to indicate delayed and normal gastric retention, respectively, were predictive of the 4-h gold-standard results, with an overall accuracy of $96.6 \%$ (6). In a separate validation cohort of 158 patients, they obtained an accuracy of $94.9 \%$. In both cohorts, use of the new criteria would have shortened the GES study to $2 \mathrm{~h}$ in approximately $80 \%$ of cases.

To further validate the Bonta criteria, we have applied the criteria to a larger group of patients aggregated from 4 North American medical centers, thereby evaluating whether the 4-h protocol can be shortened without a significant impact on the test accuracy.

\section{MATERIALS AND METHODS}

\section{Patient Population}

The population of this multicenter study included 431 patients, with approximately $25 \%$ derived from each of 4 sites (Table 1): The Ottawa Hospital, Ottawa, Ontario, Canada; Montefiore Medical Center, Bronx, New York; University of Vermont Hospital, Burlington, Vermont; and Baylor Scott \& White Health, Central Texas, Temple, Texas. All patients were imaged according to the Society of Nuclear Medicine and Molecular Imaging protocol (5). Briefly, patients ingested a meal consisting of $120 \mathrm{~mL}(4 \mathrm{oz})$ of liquid egg whites admixed with $18.5-37 \mathrm{MBq}(0.5-1 \mathrm{mCi})$ of ${ }^{99 \mathrm{~m}} \mathrm{Tc}$-sulfur colloid, 2 slices of toasted white bread, $30 \mathrm{~g}$ of jam, and $120 \mathrm{~mL}$ of water. Anterior and posterior planar images centered on the upper abdomen 
TABLE 1

Patient Characteristics for Each Center

\begin{tabular}{lcrr}
\hline \multicolumn{1}{c}{ Center } & $n$ & Women $(n)$ & Delayed 4-h emptying $(n)$ \\
\hline Montefiore Medical Center & 130 & $89(68.5 \%)$ & $34(26.2 \%)$ \\
Baylor Scott \& White Health & 100 & $74(74.0 \%)$ & $23(23.0 \%)$ \\
Ottawa Hospital & 101 & $77(76.2 \%)$ & $17(16.8 \%)$ \\
University of Vermont Hospital & 100 & $66(66.0 \%)$ & $31(31.0 \%)$ \\
Total & 431 & $306(71.0 \%)$ & $105(24.4 \%)$ \\
\hline
\end{tabular}

were acquired, and gastric activity was calculated using geometric mean (square root of the product of counts in the anterior and posterior regions of interest). Imaging was performed hourly over $4 \mathrm{~h}$, unless gastric retention was equal to or less than $10 \%$, whereupon imaging was terminated. Only patients who consumed the whole standardized meal were included in the study. This retrospective study was approved by The Ottawa Hospital Research Ethic Board as well as Institutional Review Boards at each medical center, and the requirement to obtain informed consent was waived.

\section{Data Analysis}

Sensitivity, specificity, and accuracy of gastric emptying based on the Bonta 2-h criteria were computed using 4-h gastric retention values greater than $10 \%$ as the standard for delayed gastric emptying. Comparisons of mean were conducted using the Student $t$ test. Comparison of the 4 sites' population characteristics was performed using the $\chi^{2}$ test. $P$ values of less than 0.05 were considered statistically significant. Results are presented as mean \pm SD. Statistical analyses were performed using Prism, version 6.01 (GraphPad Software), for Windows (Microsoft).

\section{RESULTS}

A total of 431 patients were included. Patient characteristics for each center are presented in Table 1. The proportion of females $(P=0.3335)$ and the prevalence of delayed gastric

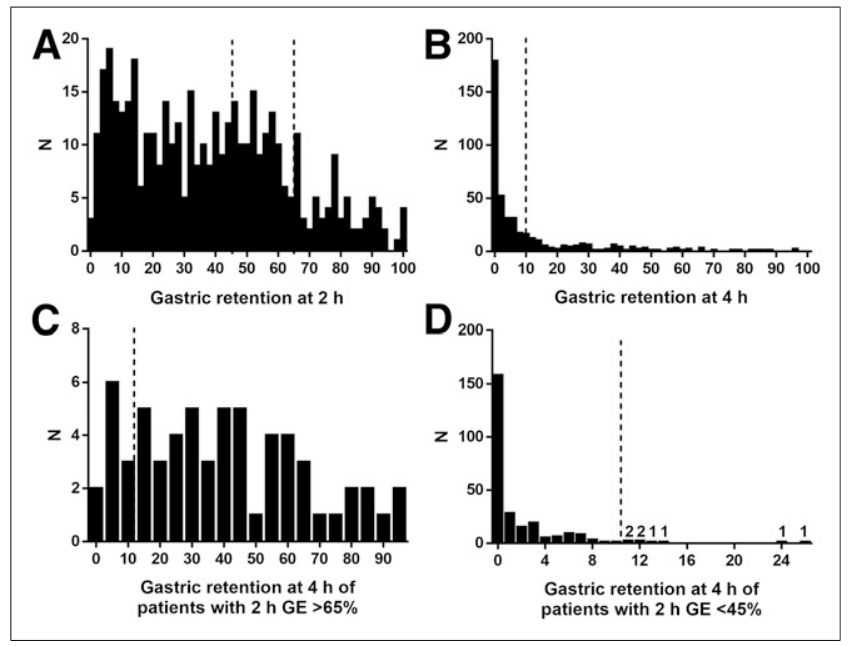

FIGURE 1. (A and B) Distribution of gastric retention values at $2 \mathrm{~h}(\mathrm{~A})$ and $4 \mathrm{~h}(\mathrm{~B})$ for all 431 participants. (C and D) Distribution of gastric retention values at $4 \mathrm{~h}$ for patients with gastric retention $>65 \%$ at $2 \mathrm{~h}$ (C) and $<45 \%$ at $2 \mathrm{~h}$ (D). emptying $(P=0.1279)$ were not significantly different among the 4 sites.

The distribution of gastric retention values at 2 and $4 \mathrm{~h}$ is shown in Figure 1. Scatterplots of the 2-h versus 4-h gastric retention values are displayed in Figure 2. The receiver-operating-characteristic curve of 2-h gastric retention (using the 4-h gastric retention values as ground truth) yielded an area under the receiveroperating-characteristic curve of 0.926 ( $P<0.0001$, Fig. 3).

\section{Bonta Criteria}

At $2 \mathrm{~h}, 261$ of 431 patients $(60.6 \%)$ had gastric retention of less than $45 \%, 62(14.4 \%)$ had gastric retention of more than $65 \%$, and $108(25.1 \%)$ had intermediate values requiring further imaging. Therefore, $323(74.9 \%)$ studies could be terminated at $2 \mathrm{~h}$ using the Bonta criteria. Overall sensitivity, specificity, and accuracy of the Bonta criteria, as applied to our multicenter cohort, were $92.4 \%, 96.9 \%$, and 95.8\%, respectively (Table 2; Fig. 3). Application of the Bonta criteria yielded $8(1.9 \%)$ false-negative and 10 (2.3\%) false-positive studies. The 4 -h gastric retention values of

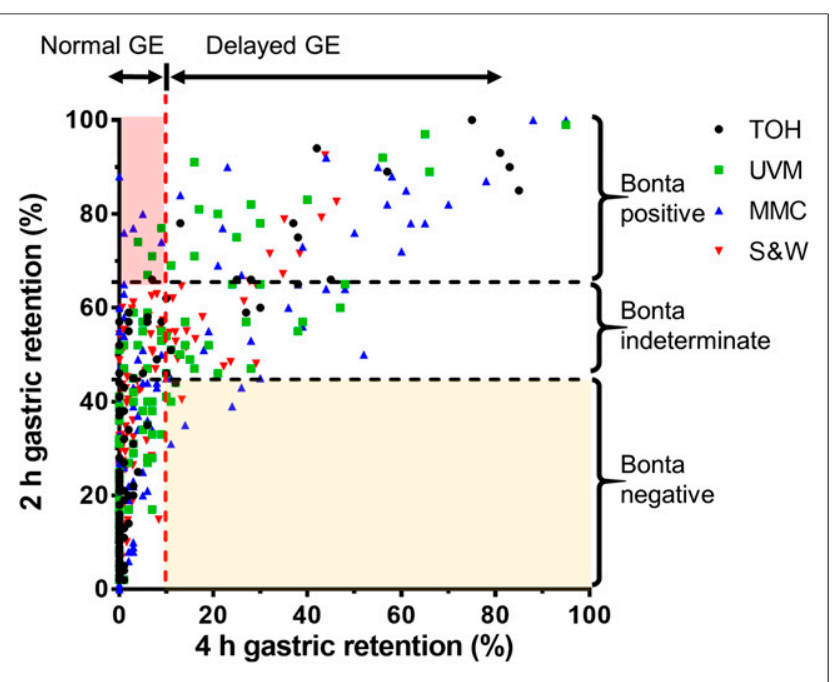

FIGURE 2. Scatterplot of 2-h vs. 4-h gastric retention values. Fourhour cutoff of $10 \%$ for delayed gastric emptying is illustrated by vertical dashed line. Bonta 2-h criteria for delayed $(>65 \%)$ and normal $(<45 \%)$ gastric retention are shown by horizontal dashed lines. False-positive results (2-h retention $>65 \%$; 4 -h retention $\leq 10 \%$ ) correspond to shaded area at upper left, whereas false-negative results (2-h retention $<45 \%$; 4-h retention $>10 \%$ ) correspond to shaded area at lower right. GE = gastric emptying; $\mathrm{MMC}=$ Montefiore Medical Center; S\&W =Baylor Scott \& White Health; $\mathrm{TOH}=$ The Ottawa Hospital; UVM = University of Vermont Hospital. 


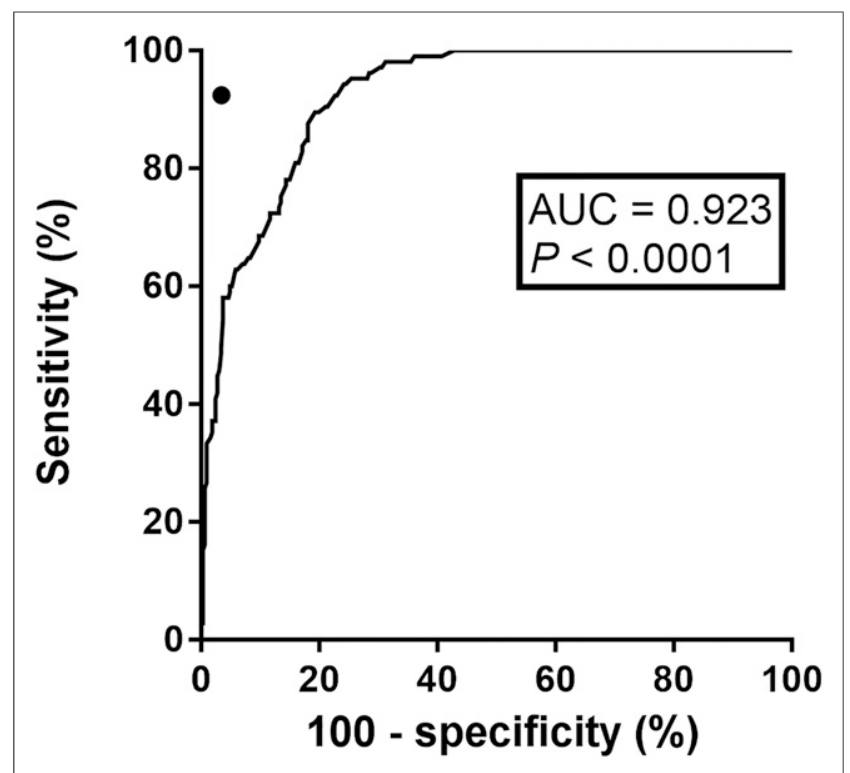

FIGURE 3. Receiver-operating-characteristic (ROC) curve for 2-h gastric retention for diagnosis of delayed 4-h gastric emptying. Sensitivity and specificity of Bonta criteria $(\bullet)$ are more accurate than any single 2-h cutoff. AUC = Area under curve.

the 8 false-negative studies were $11 \%, 11 \%, 12 \%, 12 \%, 13 \%$, $14 \%, 24 \%$, and 26\% (Fig. 1D).

\section{Number of Images}

The cumulative number of patients with gastric retention of $10 \%$ or less at $1,2,3$, and $4 \mathrm{~h}$ were $6,77,215$, and 326 , respectively. The total number of images needed to complete the study using standard criteria was 1,768 . The total number of images acquired when applying the Bonta criteria was 1,490, representing $84 \%$ of baseline. Use of the Bonta criteria resulted in a significantly shorter average per-patient imaging time of $2.46 \pm$ 0.85 versus $3.10 \pm 0.73 \mathrm{~h}(P<0.0001)$ for the standard method, a reduction of $20.6 \%$.

\section{DISCUSSION}

Bonta et al. proposed an algorithm to shorten gastric emptying imaging based on the standardized GES protocol (6). They retrospectively optimized cutoff criteria in a cohort of 174 patients and validated these criteria in a second independent cohort of 154 patients, in whom an accuracy of $94.9 \%$ was demonstrated. The purpose of the current study was to validate the Bonta criteria applied to a broader population in a multicenter study.

TABLE 2

Contingency Table of Shortened Bonta Protocol Compared with 4-Hour Retention

\begin{tabular}{lccc}
\hline \multicolumn{1}{c}{ Finding } & $4 \mathrm{~h}>10 \%$ & $4 \mathrm{~h} \leq 10 \%$ & Total \\
\hline Shortened protocol positive & 97 & 10 & 107 \\
Shortened protocol negative & 8 & 316 & 324 \\
Total & 105 & 326 & 431 \\
\hline
\end{tabular}

The population studied was similar at all 4 sites, as well as similar to the original cohorts of Bonta et al., with comparable proportions of female patients and disease prevalence (Table 3). In our multicenter cohort of patients, accuracy (95.6\%) using the shortened criteria was similar to that obtained by Bonta (94.9\%) in the validation group. The specificity (96.9\%) and accuracy $(95.8 \%)$ obtained using the shortened protocol were excellent, and superior to any single 2-h cutoff value that could be applied to the multicenter cohort (Fig. 2). On first analysis, sensitivity using the Bonta criteria (92.4\%) appears low, because application of these criteria resulted in 8 false-negative studies. In reality, 6 of these 8 patients had 4-h gastric retention of $11 \%-14 \%$ and 4 had gastric retention of $11 \%-12 \%$, which, depending on the precision of the examination, may well be indistinguishable from a normal value of $10 \%$ and unlikely to have any clinical significance. Indeed, small changes in regions of interests can lead to significant changes in gastric retention values, especially when adjacent bowel activity is present. Were we to reclassify the 4-h values of $11 \%-12 \%$ to be normal gastric retention, then the sensitivity of the examination would have been $96.0 \%$ and the corresponding accuracy $96.8 \%$. Of course, revised 2 -h criteria could be applied to the 2-h gastric retention values to improve sensitivity and specificity; however, this would also reduce the number of patients in whom the GES would be shortened (Fig. 2). We have therefore chosen to prospectively evaluate the criteria as set by Bonta rather than retrospectively choose another set of criteria that would require further independent validation, though this strategy could be pursued by individual centers. Indeed, application of these criteria over the multicenter cohort resulted in excellent results, consistent with the original findings of Bonta et al. (6).

When applying the Bonta criteria to our multicenter cohort, the study length was reduced in $55.7 \%$ of patients, whereas the total number of images required decreased by $15.7 \%$. Per-patient imaging time declined to $2.46 \mathrm{~h}$ as compared with $3.10 \mathrm{~h}$, a reduction of $20.6 \%$. These parameters translate into decreased resource use, more rapid patient discharge, and presumably improved patient satisfaction.

Shortening the gastric emptying protocol to $2 \mathrm{~h}$ in most patients, with minimal impact on test accuracy, can be convenient for patients and reduce camera time. Nevertheless, it may be prudent to weigh the advantage of the shortened test against the information lost. Finally, patients classified as normal on the basis of

TABLE 3

Comparison of Current and Bonta-Based Results (6)

\begin{tabular}{cccc}
\hline & Multicenter & $\begin{array}{c}\text { Bonta } \\
\text { original } \\
\text { dataset }\end{array}$ & $\begin{array}{c}\text { Bonta } \\
\text { validation } \\
\text { dataset }\end{array}$ \\
\hline$n \quad 431$ & 174 & 158 \\
\hline $\begin{array}{c}\text { Prevalence of } \\
\text { delayed gastric } \\
\text { emptying (\%) }\end{array}$ & 24.4 & 26 & Not applicable \\
$\begin{array}{c}\text { Proportion of } \\
\text { patients } \\
\text { stopped } \\
\text { at 2 h (\%) }\end{array}$ & 74.9 & 79 & 78 \\
\hline \begin{tabular}{c} 
Accuracy (\%) \\
\hline
\end{tabular} & 95.8 & 96.6 & \\
\hline
\end{tabular}


2-h values may actually present abnormally rapid gastric emptying, or dumping, defined as retention of less than $30 \%$ at $1 \mathrm{~h}(4,5)$. Therefore, imaging at $1 \mathrm{~h}$ remains important since it can provide additional diagnostic information.

\section{CONCLUSION}

In our application of the Bonta criteria to a multicenter cohort of patients, we have found that the criteria shortened studies in most patients and resulted in an effective compromise between preserved accuracy (95.8\%), reduced resource use, and improved patient convenience. Our results support the possibility of using these shortened criteria, or a variation thereof, in clinical practice.

\section{DISCLOSURE}

The costs of publication of this article were defrayed in part by the payment of page charges. Therefore, and solely to indicate this fact, this article is hereby marked "advertisement" in accordance with 18 USC section 1734 . No potential conflict of interest relevant to this article was reported.

\section{ACKNOWLEDGMENT}

We thank Carla Bailey of Baylor Scott \& White Health, Central Texas, Temple, for her contribution in the collection of data.

\section{REFERENCES}

1. Parkman HP, Hasler WL, Fisher RS; American Gastroenterological Association. American Gastroenterological Association medical position statement: diagnosis and treatment of gastroparesis. Gastroenterology. 2004;127:1589-1591.

2. Soykan I, Sivri B, Sarosiek I, Kiernan B, McCallum RW. Demography, clinical characteristics, psychological and abuse profiles, treatment, and long-term followup of patients with gastroparesis. Dig Dis Sci. 1998;43:2398-2404.

3. Abell TL, Camilleri M, Donohoe K, et al. Consensus Recommendations for Gastric Emptying Scintigraphy: A Joint Report of the American Neurogastroenterology and Motility Society and the Society of Nuclear Medicine. Am J Gastroenterol. 2008;103:753-763.

4. Tougas G, Eaker EY, Abell TL, et al. Assessment of gastric emptying using a low fat meal: establishment of international control values. Am J Gastroenterol. 2000;95:1456-1462.

5. Donohoe KJ, Maurer AH, Ziessman HA, et al. Procedure guideline for adult solidmeal gastric-emptying study 3.0. J Nucl Med Technol. 2009;37:196-200.

6. Bonta DV, Lee HY, Ziessman HA. Shortening the 4-hour gastric-emptying protocol. Clin Nucl Med. 2011;36:283-285. 